\title{
Theoretical Determination of Rate Constants from Excited-States: Application to Benzophenone
}

Katsuyuki Shizu, and Hironori Kaji*

Institute for Chemical Research, Kyoto University, Uji, Kyoto 611-0011, Japan

\section{KEYWORDS}

The Fermi golden rule; Rate constant, Intersystem crossing; Spin-orbit coupling; Vibronic coupling; Internal conversion; Benzophenone

This is an initial version of the manuscript submitted to The Journal of Physical Chemistry Part A (Submission date: January 23, 2021). 


\begin{abstract}
A cost-effective method of theoretically predicting electronic transition rate constants from the excited-states of molecules is reported. This method is based on density functional theory calculations of electronic states and quantitative rate constant determination with the Fermi golden rule. The method is applied to theoretical determination of the excited-state decay mechanism of photoexcited benzophenone, a representative molecule in materials science, photochemistry, and biochemistry. Calculated rate constants for benzophenone are quantitatively consistent with experimental ones, which validates the reliability of our rate constant calculation. The calculated population dynamics indicate that $S_{1} \rightarrow T_{2}\left(\leftrightarrow T_{3}\right) \rightarrow T_{1} \rightarrow S_{0}$ is the predominant decay pathway.
\end{abstract}




\section{INTRODUCTION}

Theoretical determination of excited-state decay mechanisms has drawn great attention because of its relevance to materials chemistry, photochemistry, and biological chemistry. Decay pathways in such systems usually involve competition between radiative and nonradiative transitions among different electronic, vibrational, and spin states. Radiative transitions include fluorescence and phosphorescence; nonradiative transitions include internal conversion (IC), intersystem crossing (ISC), and geometry relaxation (GR). Calculation of transition rate constants is an effective approach to theoretically determining excited-state decay paths. ${ }^{1}$ From calculated transition rate constants, one can easily determine relevant electronic transitions and rate-determining steps. Furthermore, by solving kinetic equations for excited and ground state populations, one can calculate the time evolution of electronic states and determine intermediate states relevant for predicting dominant decay paths.

When calculating transition rate constants of materials in practical use, we are faced with two questions. (1) Which quantum chemical method will accurately predict molecular properties at low computational cost? (2) Which rate expression is suitable for obtaining reliable rate constants? Although these questions have been debated for many years, there is no definitive solution and different choices have been used for different molecular systems. Density functional theory $(\mathrm{DFT})^{2}$ and time-dependent DFT (TD-DFT) ${ }^{3}$ have been widely used to predict molecular properties in solution and the solid state. DFT has been used to calculate electronic ground-state molecular geometries and ground-state properties, such as permanent dipole moments and vibrational modes, whereas TD-DFT has been used to calculate electronic excited-state properties, such as transition dipole moments (oscillator strength) and emission and absorption wavelengths. Complete active space self-consistent field (CASSCF) ${ }^{4-6}$ and multireference 
Møller-Plesset perturbation theory (MRMP) ${ }^{7-9}$ are also used for such calculations. CASSCF and MRMP are useful for calculating multiple electron excitations but have higher computational costs than TD-DFT. The purpose of this study is to theoretically calculate single electron excitations, for which TD-DFT is generally favored, especially in materials chemistry. The main advantage of DFT and TD-DFT is that by choosing a suitable functional, we can quantitatively predict molecular properties with a reasonable computational cost. We show here that all experimentally determined rate constants, radiative (fluorescence) and nonradiative decay from $S_{1}$ to $S_{0}$, radiative decay (phosphorescence) from $T_{1}$ to $S_{0}$, and ISC from $S_{1}$ to $T_{2}$, are quantitatively reproduced by cost-effective DFT and TD-DFT calculations with reasonable computation times.

Rate constants for transitions between weakly coupled states can be calculated using the Fermi golden rule. ${ }^{10}$ The general expression of the Fermi golden rule is as: $k=$ $\frac{2 \pi}{\hbar}\left\langle\Phi_{\text {init }}\left|\mathcal{H}^{\prime}\right| \Phi_{\text {fin }}\right\rangle^{2} \rho$, where $k$ denotes the rate constant for an electronic transition between initial and final states, $\left\langle\Phi_{\text {init }}\right|$ and $\left\langle\Phi_{\text {fin }}\right|$ are the wave functions of the initial and final states of a molecule, respectively, $\mathcal{H}^{\prime}$ is the perturbation operator that activates the electronic transition, and $\rho$ is the density of states. The Fermi golden rule is applicable when $\left\langle\Phi_{\text {init }}\left|\mathcal{H}^{\prime}\right| \Phi_{\text {fin }}\right\rangle$ and $\rho$ are calculated. However, calculating $\left\langle\Phi_{\text {init }}\left|\mathcal{H}^{\prime}\right| \Phi_{\text {fin }}\right\rangle$ involves integrals between vibrational wavefunctions and often cannot be performed because of the difficulty of the calculations, especially those involving nonradiative transitions. Alternatively, the semiclassical Marcus expression ${ }^{11-13}$ has been used to calculate rate constants. In the Marcus formula, $\left\langle\Phi_{\text {init }}\left|\mathcal{H}^{\prime}\right| \Phi_{\text {fin }}\right\rangle$ is replaced by the electronic coupling between the initial and final electronic states, which can be calculated more easily than $\left\langle\Phi_{\text {init }}\left|\mathcal{H}^{\prime}\right| \Phi_{\text {fin }}\right\rangle \cdot{ }^{14-15}$ However, the Marcus formula is derived from the fully quantum-mechanical Fermi golden rule by assuming the high-temperature and short- 
time limits. These assumptions make the Marcus formula invalid, especially for charge transfer transitions via nuclear tunneling, for which the Fermi golden rule is more suitable. ${ }^{16-19}$ Recently, various charge transfer excited states and electronic transitions between them (a kind of nonradiative transition) have received considerable attention in materials chemistry. To develop a method of calculating rate constants applicable to modern materials chemistry, it would be desirable to establish a cost-effective approach to predicting all relevant rate constants by the Fermi golden rule.

The aim of this study is to propose a cost-effective method for theoretically determining excited-state decay paths of molecules. DFT and TD-DFT are used to calculate molecular properties and the Fermi golden rule is used to evaluate transition rate constants. As stated above, TD-DFT and the Fermi golden rule are useful for establishing a method of calculating rate constants for applications in materials chemistry. This method is applied to analyze the excited-state decay mechanism of photoexcited benzophenone (BP), which is a representative molecule in materials chemistry, photochemistry, and biological chemistry. The excited-state decay path of BP has been subject to debate for more than 50 years. ${ }^{20-33}$ Some experimental studies on the decay mechanism of photoexcited BP have suggested that a direct $\mathrm{S}_{1} \rightarrow \mathrm{T}_{1}$ ISC is the main decay path ${ }^{23-24}$, whereas others have suggested that an indirect $\mathrm{S}_{1} \rightarrow$ intermediate states $\rightarrow \mathrm{T}_{1}$ decay path is favored over the direct $\mathrm{S}_{1} \rightarrow \mathrm{T}_{1}$ pathway. Thus, photoexcited BP is a good example for testing our method of calculating transition rate constants and corroborating the decay paths. In this study, calculated population dynamics based on the calculated transition rate constants suggest that the $S_{1}$ of photoexcited BP decays to $T_{1}$ via $T_{2}$ - and $T_{3}$-mediated decay channels, which supports experimental observations reported by Yabumoto et al. ${ }^{28}$ and Aloïse et $a l .{ }^{30}$ In addition, the transition rate constants are in good agreement with experimental results. 
Despite the low computational costs, our method can be used to predict excited-state decay paths of molecules and for quantitative predictions of their rate constants.

\section{COMPUTATIONAL METHODS}

Non-radiative and radiative transition rate constants of a molecule are calculated by the Fermi golden rule and wave functions obtained with DFT and TD-DFT methods. B3LYP/631G(d) and TD-B3LYP/6-31G(d) methods are used to calculate the electronic ground states and excited states, respectively. Nonradiative transitions between singlet states, $\mathrm{S}_{k}$ and $\mathrm{S}_{l}(k \neq l ; k, l \geq$ $0)$, occur via $S_{k-} S_{l}$ IC. The IC rate constant $\left(k_{\mathrm{IC}}\right)$ can be obtained by replacing $\mathcal{H}^{\prime}$ in the Fermi golden rule with the first-order vibronic coupling operator, $\frac{\partial \widehat{U}_{\mathrm{en}}}{\partial Q_{m}}$, where $\widehat{U}_{\mathrm{en}}$ denotes the electron-nuclear potential of the molecule and $Q_{m}$ denotes the normal coordinate of the $m^{\text {th }}$ vibrational mode. Let $k_{\mathrm{IC}, m}$ denote the rate constant for the $\mathrm{S}_{k}-\mathrm{S}_{l} \mathrm{IC}$ activated by the $m^{\text {th }}$ vibrational mode. $k_{\mathrm{IC}}$ can be written as the sum of $k_{\mathrm{IC}, m}: k_{\mathrm{IC}}=\sum_{m=1}^{M} k_{\mathrm{IC}, m}$, where $M$ is the total number of vibrational modes. $k_{\mathrm{IC}, m}$ can be expressed as ${ }^{34-38}$ :

$$
\begin{aligned}
& k_{\mathrm{IC}, m}=\frac{2 \pi \hbar^{3}}{\Delta E_{\mathrm{FC}}{ }^{2}} V_{m}{ }^{2} P_{m}, \\
& V_{m}=\left\langle S_{k}\left|\frac{\partial \widehat{U}_{\mathrm{en}}}{\partial Q_{m}}\right| \mathrm{S}_{l}\right\rangle \\
& P_{m}=\sinh \left(\frac{1}{2} \beta \hbar \omega_{m}\right) \sum_{v_{m}^{\mathrm{i}}=0}^{\infty} \exp \left\{-\left(\frac{1}{2}+v_{m}\right) \beta \hbar \omega_{m}\right\} \times\left\{v_{m} D\left(\Delta E_{\mathrm{AD}}+\hbar \omega_{m}\right)+\right. \\
& \left.\left(v_{m}+1\right) D\left(\Delta E_{\mathrm{AD}}-\hbar \omega_{m}\right)\right\},
\end{aligned}
$$

where $V_{m}$ denotes the magnitude of vibronic coupling between $\mathrm{S}_{k}$ and $\mathrm{S}_{l}$ activated by the $m^{\text {th }}$ vibrational mode, $\Delta E_{\mathrm{FC}}$ denotes the difference between the $\mathrm{S}_{0}-\mathrm{S}_{k}$ and $\mathrm{S}_{0}-\mathrm{S}_{l}$ vertical excitation 
energies ( $\mathrm{FC}$ is the abbreviation for Franck-Condon), $\Delta E_{\mathrm{AD}}$ denotes the adiabatic energy difference between $\mathrm{S}_{k}$ and $\mathrm{S}_{l}, \beta$ denotes the inverse temperature $\left(\beta^{-1}=k_{\mathrm{B}} T\right.$, where $k_{\mathrm{B}}$ is the Boltzmann constant and $T=300 \mathrm{~K}$ is the temperature, and $\omega_{m}$ and $v_{m}$ denote the angular frequency and vibrational quantum number of the $m^{\text {th }}$ vibrational mode, respectively, and $D(x)=\frac{1}{\pi} \frac{\gamma}{x^{2}+\gamma^{2}}$ denotes the Lorentzian distribution function $\left(\gamma=\mathrm{fwhm} / 2\right.$ and $\left.\mathrm{fwhm}=600 \mathrm{~cm}^{-1}\right)$ describing dephasing effects owing to interactions with surrounding molecules. ${ }^{38}$ The value of the fwhm was originally determined for a tetracene single crystal, ${ }^{39}$ which is typical value for a low molecular weight organic compound. Our calculations reproduced the experimental ISC rate constant of $\mathrm{BP}$ in its single crystal form. $\Delta E_{\mathrm{FC}}, V_{m}$, and $\omega_{m}$ were calculated for the optimized geometries. $P_{m}$ contains information regarding the density of the final vibrational states. The energy-downhill $S_{k}-S_{l}$ rate constant is first calculated as $k_{\text {IC }}$ and then, the energy-uphill $S_{k}-S_{l}$ rate constant is calculated as $k_{\mathrm{IC}} \exp \left(-\beta \Delta E_{\mathrm{FC}} / 2\right)$. The factor $1 / 2$ in the exponential function means that the position of the transition state along the reaction coordinate is halfway between the $\mathrm{S}_{k}$ and $\mathrm{T}_{l}$ geometries. ${ }^{40}$ Equations $1-3$ are also used to calculate nonradiative transition rate constants between triplet states, $\mathrm{T}_{k}$ and $\mathrm{T}_{l}(k \neq l ; k, l \geq 1)$, by replacing $\mathrm{S}_{k}$ and $\mathrm{S}_{l}$ with $\mathrm{T}_{k}$ and $\mathrm{T}_{l}$, respectively, and $\Delta E_{\mathrm{FC}}$ with the difference between the $\mathrm{S}_{0}-\mathrm{T}_{k}$ and $\mathrm{S}_{0}-\mathrm{T}_{l}$ vertical excitation energies, and $\Delta E_{\mathrm{AD}}$ with the adiabatic energy difference between $\mathrm{T}_{k}$ and $\mathrm{T}_{l}$.

$\mathrm{S}_{k}-\mathrm{T}_{l}(k \geq 0, l \geq 1)$ nonradiative transition occurs via $\mathrm{S}_{k}-\mathrm{T}_{l}$ ISC. By replacing $\mathcal{H}^{\prime}$ in the Fermi golden rule with a one-electron spin-orbit coupling operator ${ }^{41}\left(\mathcal{H}_{\mathrm{SOC}}\right)$, the ISC rate constant $\left(k_{\mathrm{ISC}, M \mathrm{~S}}\right)$ for the three degenerated triplet sublevels $\mathrm{T}_{l}^{M_{\mathrm{S}}}\left(M_{\mathrm{S}}=0, \pm 1\right)$ can be written as

$$
k_{\mathrm{ISC}, M_{\mathrm{S}}}=\frac{2 \pi}{\hbar}\left|\left\langle\mathrm{S}_{k}\left|\mathcal{H}_{\mathrm{SOC}}\right| \mathrm{T}_{l}^{M_{\mathrm{S}}}\right\rangle\right|^{2} D\left(\Delta E_{\mathrm{AD}}\right)
$$


where $\Delta E_{\mathrm{AD}}$ denotes the adiabatic energy difference between $\mathrm{S}_{k}$ and $\mathrm{T}_{l}$. When the $\mathrm{S}_{k} \rightarrow \mathrm{T}_{l}$ nonradiative transition is a downhill energy transition, the $\mathrm{S}_{k} \rightarrow \mathrm{T}_{l}^{M_{\mathrm{S}}}$ rate constant is expressed as $k_{\mathrm{ISC}, M_{\mathrm{S}}}$, whereas the energy-uphill $\mathrm{T}_{l}^{M_{\mathrm{S}}} \rightarrow \mathrm{S}_{k}$ rate constant is expressed as $k_{\mathrm{ISC}, M_{\mathrm{S}}} \exp \left(-\beta \Delta E_{\mathrm{FC}} / 2\right)$. Hence, the rate equations for $\mathrm{S}_{k}$ and $\mathrm{T}_{l}$ can be written as

$$
\begin{aligned}
& \frac{d\left[\mathrm{~S}_{k}\right]}{d t}=-\left(\sum_{M_{\mathrm{S}}=0, \pm 1} k_{\mathrm{ISC}, M_{\mathrm{S}}}\right)\left[\mathrm{S}_{k}\right]+\sum_{M_{\mathrm{S}}=0, \pm 1} k_{\mathrm{ISC}, M_{\mathrm{S}}} \exp \left(-\beta \Delta E_{\mathrm{FC}} / 2\right)\left[\mathrm{T}_{l}^{M_{\mathrm{S}}}\right]+A \\
& \frac{d\left[\mathrm{~T}_{l}\right]}{d t}=\left(\sum_{M_{\mathrm{S}}=0, \pm 1} k_{\mathrm{ISC}, M_{\mathrm{S}}}\right)\left[\mathrm{S}_{k}\right]-\sum_{M_{\mathrm{S}}=0, \pm 1} k_{\mathrm{ISC}, M_{\mathrm{S}}} \exp \left(-\beta \Delta E_{\mathrm{FC}} / 2\right)\left[\mathrm{T}_{l}^{M_{\mathrm{S}}}\right]+B \\
& {\left[\mathrm{~T}_{l}\right]=\sum_{M_{\mathrm{S}}=0, \pm 1}\left[\mathrm{~T}_{l}^{M_{\mathrm{S}}}\right],}
\end{aligned}
$$

where $\left[\mathrm{S}_{k}\right],\left[\mathrm{T}_{l}\right]$, and $\left[\mathrm{T}_{l}^{M_{\mathrm{S}}}\right]$ denote the respective populations of $\mathrm{S}_{k}, \mathrm{~T}_{l}$, and $\mathrm{T}_{l}^{M_{\mathrm{S}}}, A$ denotes the contributions from the electronic states other than $\left[\mathrm{S}_{k}\right]$ and $\left[\mathrm{T}_{l}^{M_{\mathrm{S}}}\right]$ to $d\left[\mathrm{~S}_{k}\right] / d t$, and $B$ denotes those to $d\left[\mathrm{~T}_{l}\right] / d t . A$ and $B$ for individual combinations of $\mathrm{S}_{k}$ and $\mathrm{T}_{l}$ are described in detail in the Supporting Information [eqs. (1)-(20)]. We reasonably assume that $\mathrm{T}_{l}^{M_{\mathrm{S}}}$ thermally equilibrates before $\mathrm{T}_{l} \rightarrow \mathrm{S}_{k}$ ISC, that is, $\left[\mathrm{T}_{l}^{M_{\mathrm{S}}}\right]=\left[\mathrm{T}_{l}\right] / 3$ holds, Equations 5 and 6 can be expressed as

$$
\begin{aligned}
& \frac{d\left[\mathrm{~S}_{k}\right]}{d t}=-\left(\sum_{M_{\mathrm{S}}=0, \pm 1} k_{\mathrm{ISC}, M_{\mathrm{S}}}\right)\left[\mathrm{S}_{k}\right]+\frac{1}{3}\left(\sum_{M_{\mathrm{S}}=0, \pm 1} k_{\mathrm{ISC}, M_{\mathrm{S}}}\right) \exp \left(-\beta \Delta E_{\mathrm{FC}} / 2\right)\left[\mathrm{T}_{l}\right]+A \\
& \frac{d\left[\mathrm{~T}_{l}\right]}{d t}=\left(\sum_{M_{\mathrm{S}}=0, \pm 1} k_{\mathrm{ISC}, M_{\mathrm{S}}}\right)\left[\mathrm{S}_{k}\right]-\frac{1}{3}\left(\sum_{M_{\mathrm{S}}=0, \pm 1} k_{\mathrm{ISC}, M_{\mathrm{S}}}\right) \exp \left(-\beta \Delta E_{\mathrm{FC}} / 2\right)\left[\mathrm{T}_{l}\right]+B .
\end{aligned}
$$

Let $k_{\mathrm{ISC}}$ denote the sum of $k_{\mathrm{ISC}, M_{\mathrm{S}}}$,

$$
k_{\mathrm{ISC}}=\sum_{M_{\mathrm{S}}=0, \pm 1} k_{\mathrm{ISC}, M_{\mathrm{S}}}
$$

Then, substituting Equation 10 into Equations 8 and 9 gives 


$$
\begin{aligned}
& \frac{d\left[\mathrm{~S}_{k}\right]}{d t}=-k_{\mathrm{ISC}}\left[\mathrm{S}_{k}\right]+\frac{k_{\mathrm{ISC}}}{3} \exp \left(-\beta \Delta E_{\mathrm{FC}} / 2\right)\left[\mathrm{T}_{l}\right]+A, \\
& \frac{d\left[\mathrm{~T}_{l}\right]}{d t}=k_{\mathrm{ISC}}\left[\mathrm{S}_{k}\right]-\frac{k_{\mathrm{ISC}}}{3} \exp \left(-\beta \Delta E_{\mathrm{FC}} / 2\right)\left[\mathrm{T}_{l}\right]+B
\end{aligned}
$$

Equations 11 and 12 show that $k_{\text {ISC }}$ represents the energy-downhill $\mathrm{S}_{k} \rightarrow \mathrm{T}_{l}$ rate constant, whereas $1 / 3 k_{\mathrm{ISC}} \exp \left(-\beta \Delta E_{\mathrm{FC}} / 2\right)$ represents the energy-uphill $\mathrm{T}_{l} \rightarrow \mathrm{S}_{k}$ rate constant. This process is frequently called reverse ISC (RISC). The prefactor $1 / 3$ reflects the thermally equilibrated triplet sublevels and appears in the $\mathrm{RISC}^{42-43}$ and phosphorescence. ${ }^{44-46}$ The factor $1 / 2$ in the exponential function means that the position of the transition state along the reaction coordinate is halfway between the $\mathrm{S}_{k}$ and $\mathrm{T}_{l}$ geometries. ${ }^{40}$ In a similar manner, when the $\mathrm{S}_{k} \rightarrow \mathrm{T}_{l}$ nonradiative transition is an energy-uphill transition, $k_{\mathrm{ISC}} \exp \left(-\beta \Delta E_{\mathrm{FC}} / 2\right)$ represents the energy-uphill $\mathrm{S}_{k} \rightarrow \mathrm{T}_{l}$ ISC rate constant, whereas $1 / 3 k_{\mathrm{ISC}}$ represents the energy-downhill $\mathrm{T}_{l} \rightarrow$ $\mathrm{S}_{k}$ RISC rate constant. One can define the effective $\mathrm{S}_{k}-\mathrm{T}_{l}$ SOC constant as:

$$
|\mathrm{SOC}|=\sqrt{\sum_{M_{\mathrm{S}}=0, \pm 1}\left|\left\langle\mathrm{~S}_{k}\left|\mathcal{H}_{\mathrm{SOC}}\right| \mathrm{T}_{l}^{M_{\mathrm{S}}}\right\rangle\right|^{2}}
$$

From Equations 4,10 , and $13, k_{\text {ISC }}$ is expressed in terms of $|\mathrm{SOC}|$ as

$$
k_{\mathrm{ISC}}=\frac{2 \pi}{\hbar}|\mathrm{SOC}|^{2} D\left(\Delta E_{\mathrm{AD}}\right)
$$

Thus, $|\mathrm{SOC}|$ represents the strength of the $\mathrm{S}_{k}-\mathrm{T}_{l} \mathrm{SOC}$ when $\mathrm{T}_{l}^{M_{\mathrm{S}}}$ is thermally equilibrated.

The $S_{1}-S_{0}$ radiative transition (fluorescence) occurs owing to interactions between the $S_{1}$ $\mathrm{S}_{0}$ transition dipole moment $\left(\mu_{\mathrm{f}, 10}\right)$ of a molecule and the electromagnetic field. From the Fermi golden rule, the fluorescence rate constant $\left(k_{\mathrm{f}}\right)$ can be expressed as $k_{\mathrm{f}}=\frac{4 \Delta E_{\mathrm{AD}}{ }^{3}}{3 \hbar^{3} c^{3}} \mu_{\mathrm{f}, 10}^{2},{ }^{47}$ where $\Delta E_{\mathrm{AD}}$ is the $\mathrm{S}_{0}-\mathrm{S}_{1}$ adiabatic energy difference and $c$ is the speed of light. The $\mathrm{T}_{1}-\mathrm{S}_{0}$ radiative transition (phosphorescence) occurs owing to interactions between the $\mathrm{T}_{1}-\mathrm{S}_{0}$ transition dipole 
moment $\left(\mu_{\mathrm{p}, 10}\right)$ and the electromagnetic field. The phosphorescence rate constant $\left(k_{\mathrm{p}}\right)$ can be expressed as $k_{\mathrm{p}}=\frac{4 \Delta E_{\mathrm{AD}}{ }^{3}}{3 \hbar^{3} c^{3}} \mu_{\mathrm{p}, 10}^{2},{ }^{46}$ where $\Delta E_{\mathrm{AD}}$ is the $\mathrm{S}_{0}-\mathrm{T}_{1}$ adiabatic energy difference.

\section{RESULTS AND DISCUSSION}

Geometry optimization and frequency analysis for $\mathrm{S}_{0}$ were performed at the B3LYP/6$31 \mathrm{G}(\mathrm{d})$ level of theory, whereas those for $\mathrm{S}_{1}, \mathrm{~T}_{1}, \mathrm{~T}_{2}$, and $\mathrm{T}_{3}$ were performed at the TD-B3LYP/6$31 \mathrm{G}(\mathrm{d})$ level of theory. Because no imaginary modes were obtained for the optimized $\mathrm{S}_{0}, \mathrm{~S}_{1}, \mathrm{~T}_{1}$, and $\mathrm{T}_{3}$ geometries, the optimized geometries were confirmed to be local energy minima of $\mathrm{S}_{0}, \mathrm{~S}_{1}$, $T_{1}$, and $T_{3}$. The geometry optimization of $T_{2}$ resulted in the equilibrium $T_{1}$ geometry. Next, the excitation energies for $S_{1}-S_{6}$ and $T_{1}-T_{6}$ at the $S_{0}, S_{1}, T_{1}$, and $T_{3}$ geometries were calculated using the TD-B3LYP/6-31G(d) method (Table S10 and Figure S3). Because $\mathrm{S}_{k}(k \geq 2)$ and $\mathrm{T}_{l}(l \geq 4)$ were found to be energetically very high $\left(>460 \mathrm{meV}\right.$ ) compared with $\mathrm{S}_{1}$ and $\mathrm{T}_{1}-\mathrm{T}_{3}$ from the TDDFT calculations, only $S_{0}, S_{1}$, and $T_{1}-T_{3}$ are considered in the following discussion. $S_{1}$ is expressed as ${ }^{1}\left(\mathrm{n}, \pi^{*}\right) l^{1}\left(\pi, \pi^{*}\right) ; \mathrm{T}_{1}$ and $\mathrm{T}_{2}$ are expressed as ${ }^{3}\left(\mathrm{n}, \pi^{*}\right) /^{\beta}\left(\pi, \pi^{*}\right)$; and $\mathrm{T}_{3}$ is expressed as ${ }^{3}\left(\pi, \pi^{*}\right) /^{3}\left(\mathrm{n}, \pi^{*}\right)$. Here, the notation ${ }^{1}\left(\mathrm{n}, \pi^{*}\right) /^{1}\left(\pi, \pi^{*}\right)$ means that $\mathrm{n}, \pi^{*}$ and $\pi, \pi^{*}$ excitations gave major and minor contributions to $\mathrm{S}_{1}$, respectively. In the same way, ${ }^{3}\left(\mathrm{n}, \pi^{*}\right) /^{\beta}\left(\pi, \pi^{*}\right)$ means that $\mathrm{n}$ $\pi^{*}$ and $\pi-\pi^{*}$ excitations make major and minor contributions, respectively, to $\mathrm{T}_{1}$ and $\mathrm{T}_{2}$. Figure 1 shows an atom labeling scheme and the calculated carbonyl $\mathrm{C}=\mathrm{O}$ bond length $\left(R_{\mathrm{C}=0}\right)$, and $\mathrm{C}_{2}-\mathrm{C}_{1}$ $\mathrm{C}_{1}{ }^{\prime}-\mathrm{C}_{2}{ }^{\prime}$ dihedral angle $(\phi)$ for the optimized $\mathrm{S}_{0}, \mathrm{~S}_{1}, \mathrm{~T}_{1}$, and $\mathrm{T}_{3}$ geometries of BP. For the $\mathrm{S}_{0}, \mathrm{~S}_{1}, \mathrm{~T}_{1}$ $\left(=\mathrm{T}_{2}\right)$, and $\mathrm{T}_{3}$ geometries, $R_{\mathrm{C}=\mathrm{O}}$ values were calculated to be $1.23,1.32,1.33$, and $1.25 \AA$, respectively, and $\phi$ as $50^{\circ}, 40^{\circ}, 45^{\circ}$, and $39^{\circ}$, respectively. Bond lengths other than the $\mathrm{C}=\mathrm{O}$ bond are shown in Figure $\mathrm{S} 1$ in Supporting Information. Figure $\mathrm{S} 1$ also compares the $\mathrm{T}_{1}$ geometries 
obtained with TD-B3LYP and UB3LYP methods. The calculated bond lengths and $\phi$ of the two geometries were almost identical, suggesting that both the UB3LYP and TD-B3LTP methods can be used to optimize the $\mathrm{T}_{1}$ geometry of BP. Figure 2 shows state energy diagrams for BP at the $S_{0}, S_{1}, T_{1}\left(\right.$ and $\left.T_{2}\right)$, and $T_{3}$ geometries. The $S_{1}$ and $T_{1}$ geometries have similar energy diagrams, which are unlike the diagrams of the $S_{0}$ and $T_{3}$ geometries. At the $S_{0}$ and $T_{3}$ geometries, $\mathrm{T}_{2}$ and $\mathrm{T}_{3}$ energy levels are close, whereas they are well separated $(>500 \mathrm{meV})$ at the $S_{1}$ and $T_{1}$ geometries. $S_{1}$ is located higher than $T_{3}$ in the $S_{0}$ and $T_{3}$ geometries, whereas it is located between $T_{2}$ and $T_{3}$ in the $S_{1}$ and $T_{1}$ geometries. Thus, the energy level alignment of $S_{1}$, $\mathrm{T}_{2}$, and $\mathrm{T}_{3}$ is affected by the molecular geometry. Calculated $|\mathrm{SOC}|$ values (Equation 13) between $\mathrm{S}_{1}, \mathrm{~T}_{1}, \mathrm{~T}_{2}$, and $\mathrm{T}_{3}$ are also shown in Figure 2. The $\left|\left\langle\mathrm{S}_{k}\left|\mathcal{H}_{\mathrm{SOC}}\right| \mathrm{T}_{l}^{M_{\mathrm{S}}}\right\rangle\right|$ values are listed in Table S12 in Supporting Information. In contrast to the excited-state energy level alignment, the $|\mathrm{SOC}|$ values decrease in the order of $\mathrm{S}_{0}-\mathrm{T}_{1}>\mathrm{S}_{1}-\mathrm{T}_{2}>\mathrm{S}_{1}-\mathrm{T}_{1}>\mathrm{S}_{1}-\mathrm{T}_{3}$ irrespective of the molecular geometry. Because $S_{1}, T_{1}, T_{2}$, and $T_{3}$ have both $\left(n, \pi^{*}\right)$ and $\left(\pi, \pi^{*}\right)$ character, the $S_{1}-T_{1}, S_{1}-T_{2}$, and $\mathrm{S}_{1}-\mathrm{T}_{3}$ SOCs are non-zero owing to the ${ }^{1}\left(\mathrm{n}, \pi^{*}\right)-^{3}\left(\pi, \pi^{*}\right)$ transitions. ${ }^{48}$ 


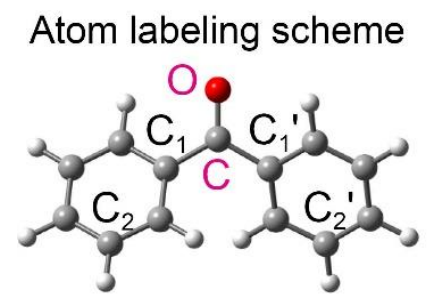

$\begin{array}{lll} & R_{\mathrm{C}=\mathrm{O}} & \phi \\ \mathrm{S}_{0} \text { geom } & 1.23 & 50 \\ \mathrm{~S}_{1} \text { geom } & 1.32 & 40 \\ \mathrm{~T}_{1} \text { geom } & 1.33 & 45 \\ \mathrm{~T}_{3} \text { geom } & 1.25 & 39\end{array}$

Figure 1. An atom labeling scheme and the calculated carbonyl $\mathrm{C}=\mathrm{O}$ bond length $\left(R_{\mathrm{C}=\mathrm{O}}\right)$ and $\mathrm{C}_{2}-\mathrm{C}_{1}-\mathrm{C}_{1}{ }^{\prime}-\mathrm{C}_{2}{ }^{\prime}$ dihedral angle $(\phi)$ for the optimized $\mathrm{S}_{0}, \mathrm{~S}_{1}, \mathrm{~T}_{1}$, and $\mathrm{T}_{3}$ geometries of $\mathrm{BP} . R_{\mathrm{C}=\mathrm{O}}$ are in $\AA$ and $\phi$ are in degrees. 


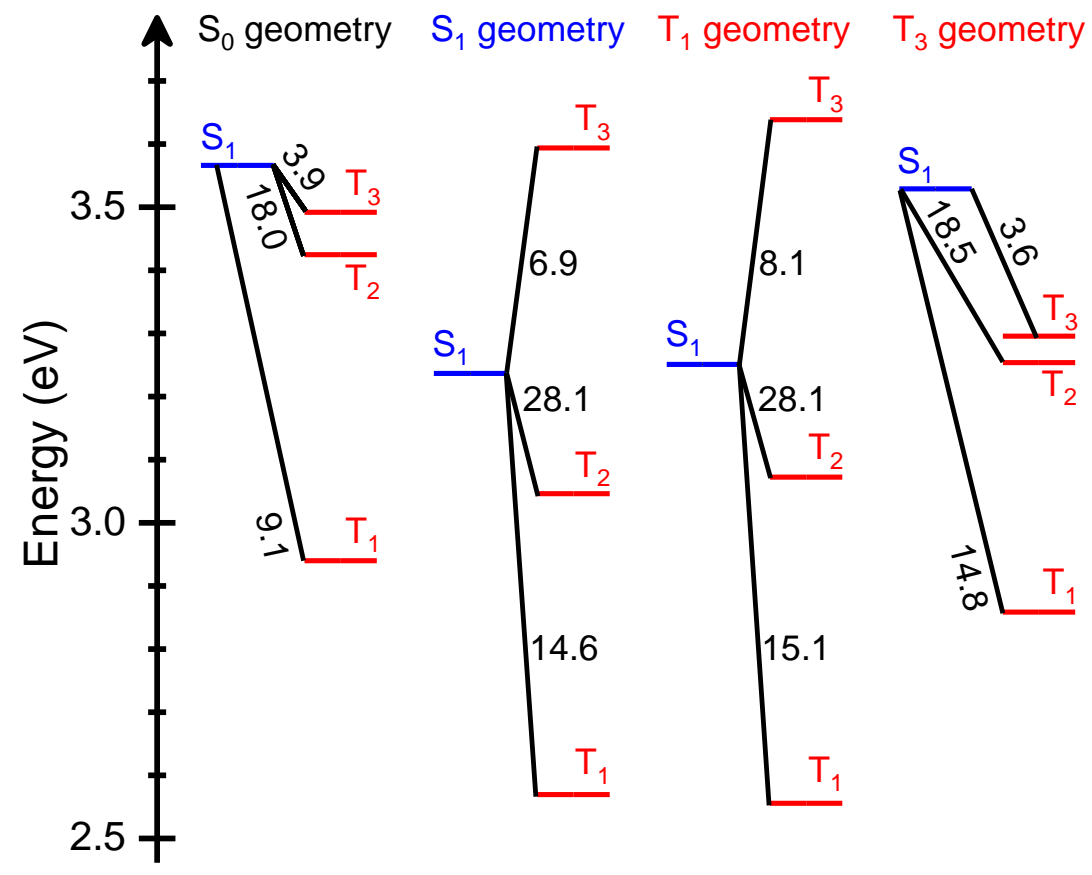

Figure 2. Energy-level diagrams of benzophenone. Numbers are $|\mathrm{SOC}|$ (Equation 13) between $\mathrm{S}_{1}, \mathrm{~T}_{1}, \mathrm{~T}_{2}$, and $\mathrm{T}_{3}$ in $\mathrm{cm}^{-1}$. Numerical values of the electronic energies are listed in Table S11. The zero point of the electronic energies is set to be the $\mathrm{S}_{0}$ energy calculated at the optimized $\mathrm{S}_{0}$ geometry. 
Radiative and nonradiative transition rate constants between $S_{0}, S_{1}, T_{1}, T_{2}$, and $T_{3}$ calculated for the $S_{0}, S_{1}, T_{1}$, and $T_{3}$ geometries are listed in Table 1. Irrespective of the molecular geometry, the $S_{1} \rightarrow T_{2}$ ISC is the fastest ISC among the ISCs from $S_{1}$ because of the large $S_{1}-T_{2}$ SOCs and energetically downhill transition. The calculated $S_{1} \rightarrow T_{2}$ ISC rate constants for the $S_{1}$ and $\mathrm{T}_{1}$ geometries $\left(3.6-3.7 \times 10^{10} \mathrm{~s}^{-1}\right)$ are in excellent agreement with the experimental value for

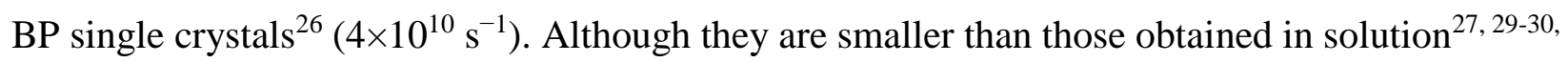
${ }^{32-33}\left(6 \times 10^{10}-1.5 \times 10^{11} \mathrm{~s}^{-1}\right)$, the overall agreement between the experimental and calculated values is reasonable. At the $\mathrm{S}_{0}$ and $\mathrm{T}_{3}$ geometries, the $\mathrm{S}_{1} \rightarrow \mathrm{T}_{3}$ ISC is an energetically downhill transition. Hence, the $S_{1} \rightarrow T_{3}$ ISC competes with the $S_{1} \rightarrow T_{2}$ ISC although the $S_{1}-T_{3} S O C$ (4.0 or $\left.3.7 \mathrm{~cm}^{-1}\right)$ is approximately one-fifth of the $\mathrm{S}_{1}-\mathrm{T}_{2} \mathrm{SOC}\left(18.0\right.$ or $\left.18.5 \mathrm{~cm}^{-1}\right)$. In contrast, at the $\mathrm{S}_{1}$ and $\mathrm{T}_{1}$ geometries, the $\mathrm{S}_{1} \rightarrow \mathrm{T}_{3}$ ISC is an energetically uphill transition and therefore, the $\mathrm{S}_{1}$ $\rightarrow \mathrm{T}_{3}$ ISC $\left(1.7\right.$ or $\left.1.3 \times 10^{7} \mathrm{~s}^{-1}\right)$ occurs much slower than the $\mathrm{S}_{1} \rightarrow \mathrm{T}_{2}$ ISC $\left(3.6\right.$ or $\left.3.7 \times 10^{10} \mathrm{~s}^{-1}\right)$. The calculated $\mathrm{S}_{1} \rightarrow \mathrm{S}_{0}$ radiative and nonradiative decay rates are of the order of $10^{6} \mathrm{~s}^{-1}$ and the calculated $\mathrm{T}_{1} \rightarrow \mathrm{S}_{0}$ radiative decay rates are of the order of $10^{2} \mathrm{~s}^{-1}$, irrespective of the molecular geometry. These values are in good agreement with the experimentally obtained values of $10^{6} \mathrm{~s}^{-1}$ for $\mathrm{S}_{1} \rightarrow \mathrm{S}_{0}$ radiative decay, $<10^{7} \mathrm{~s}^{-1}$ for $\mathrm{S}_{1} \rightarrow \mathrm{S}_{0}$ nonradiative decay, and $1.5 \times 10^{2} \mathrm{~s}^{-1}$ for $\mathrm{T}_{1} \rightarrow$ $\mathrm{S}_{0}$ radiative decay. ${ }^{1,20-21}$ 
Table 1. Nonradiative and radiative transition rate constants $\left(\mathrm{s}^{-1}\right)$ calculated for the $\mathrm{S}_{0}, \mathrm{~S}_{1}, \mathrm{~T}_{1}$, and $\mathrm{T}_{3}$ geometries of $\mathrm{BP}$.

\begin{tabular}{|c|c|c|c|c|c|}
\hline & $\mathrm{S}_{0}$ geom & $\mathrm{S}_{1}$ geom & $\mathrm{T}_{1}$ geom & $\mathrm{T}_{3}$ geom & Expt. \\
\hline \multicolumn{6}{|l|}{ Nonradiative transition (ISC) } \\
\hline $\mathrm{S}_{1} \rightarrow \mathrm{T}_{1}$ & $3.1 \times 10^{8}$ & $8.0 \times 10^{8}$ & $8.6 \times 10^{8}$ & $8.2 \times 10^{8}$ & \multirow{7}{*}{$4 \times 10^{10 *}$} \\
\hline $\mathrm{S}_{1} \rightarrow \mathrm{T}_{2}$ & $1.5 \times 10^{10}$ & $3.6 \times 10^{10}$ & $3.7 \times 10^{10}$ & $1.6 \times 10^{10}$ & \\
\hline $\mathrm{S}_{1} \rightarrow \mathrm{T}_{3}$ & $5.6 \times 10^{9}$ & $1.7 \times 10^{7}$ & $1.3 \times 10^{7}$ & $4.8 \times 10^{9}$ & \\
\hline $\mathrm{T}_{1} \rightarrow \mathrm{S}_{0}$ & $1.8 \times 10^{8}$ & $2.0 \times 10^{8}$ & $2.1 \times 10^{8}$ & $1.1 \times 10^{8}$ & \\
\hline $\mathrm{T}_{1} \rightarrow \mathrm{S}_{1}$ & $5.6 \times 10^{2}$ & $6.6 \times 10^{2}$ & $4.1 \times 10^{2}$ & $6.4 \times 10^{2}$ & \\
\hline $\mathrm{T}_{2} \rightarrow \mathrm{S}_{1}$ & $3.2 \times 10^{8}$ & $3.0 \times 10^{8}$ & $3.9 \times 10^{8}$ & $2.6 \times 10^{7}$ & \\
\hline $\mathrm{T}_{3} \rightarrow \mathrm{S}_{1}$ & $4.4 \times 10^{8}$ & $5.7 \times 10^{9}$ & $7.9 \times 10^{9}$ & $1.8 \times 10^{7}$ & \\
\hline \multicolumn{6}{|l|}{ Nonradiative transition (IC) } \\
\hline $\mathrm{S}_{1} \rightarrow \mathrm{S}_{0}$ & $2.3 \times 10^{6}$ & $2.0 \times 10^{6}$ & $2.0 \times 10^{6}$ & $2.2 \times 10^{6}$ & \multirow[t]{5}{*}{$<10^{7}$} \\
\hline $\mathrm{T}_{1} \rightarrow \mathrm{T}_{2}$ & $8.5 \times 10^{5}$ & $7.0 \times 10^{5}$ & $2.7 \times 10^{5}$ & $9.8 \times 10^{6}$ & \\
\hline $\mathrm{T}_{2} \rightarrow \mathrm{T}_{1}$ & $1.1 \times 10^{10}$ & $7.1 \times 10^{9}$ & $5.9 \times 10^{9}$ & $2.0 \times 10^{10}$ & \\
\hline $\mathrm{T}_{2} \rightarrow \mathrm{T}_{3}$ & $2.0 \times 10^{12}$ & $2.0 \times 10^{6}$ & $9.6 \times 10^{5}$ & $2.0 \times 10^{12}$ & \\
\hline $\mathrm{T}_{3} \rightarrow \mathrm{T}_{2}$ & $7.5 \times 10^{12}$ & $8.1 \times 10^{10}$ & $5.5 \times 10^{10}$ & $4.4 \times 10^{12}$ & \\
\hline \multicolumn{6}{|l|}{ Radiative decay } \\
\hline $\mathrm{S}_{1} \rightarrow \mathrm{S}_{0}$ (fluorescence) & $4.5 \times 10^{6}$ & $3.3 \times 10^{6}$ & $4.2 \times 10^{6}$ & $3.7 \times 10^{6}$ & $10^{6}$ \\
\hline $\mathrm{T}_{1} \rightarrow \mathrm{S}_{0}$ (phosphorescence) & $1.9 \times 10^{2}$ & $5.1 \times 10^{2}$ & $5.8 \times 10^{2}$ & $2.9 \times 10^{2}$ & $1.5 \times 10^{2}$ \\
\hline
\end{tabular}

To determine decay paths of photoexcited BP, we calculated the time evolution of the $\mathrm{S}_{0}$, $\mathrm{S}_{1}, \mathrm{~T}_{1}, \mathrm{~T}_{2}$, and $\mathrm{T}_{3}$ populations. Hereafter, if necessary, $\mathrm{S}_{0}, \mathrm{~S}_{1}, \mathrm{~T}_{1}, \mathrm{~T}_{2}$, and $\mathrm{T}_{3}$ are written in the form [electronic state]@ [optimized geometry] to distinguish each electronic state at each optimized geometry: for example, $\mathrm{S}_{1} @ \mathrm{~S}_{0}$ denotes the $\mathrm{S}_{1}$ state for the $\mathrm{S}_{0}$ geometry. Because we intended to reveal the decay channel from $\mathrm{S}_{1} @ \mathrm{~S}_{0}$ generated by photoexcitation of the ground state $\left(\mathrm{S}_{0} @ \mathrm{~S}_{0}\right)$, the initial population of $\mathrm{S}_{1} @ \mathrm{~S}_{0}$ was set to be 1 and those of the other states were set to be 0 . Note that the total sum of the populations was always 1 at any time point because neither fission nor fusion between electronic states were involved in our population analysis. 
Geometry relaxations in $\mathrm{S}_{0}, \mathrm{~S}_{1}, \mathrm{~T}_{1}, \mathrm{~T}_{2}$, and $\mathrm{T}_{3}$ can compete with electronic transitions. As stated above, the geometry relaxations in $\mathrm{T}_{2}$ is eventually deform the molecular geometry to the optimized $\mathrm{T}_{1}$ geometry. The rate constants of the geometry relaxations $\left(k_{\mathrm{GR}}\right)$ were unknown and set to a uniform value. From the electronic-transition rate constants and $k_{\mathrm{GR}}$, one can obtain kinetic equations to be solved for calculating the time evolution of the populations (Eqs S1-20 and Table 13 in Supporting Information). The kinetic equations were solved numerically with a time step of 5 fs.

First, we examine decay paths from $\mathrm{S}_{1} @ \mathrm{~S}_{0}$ without any geometry relaxation $\left(k_{\mathrm{GR}}=0 \mathrm{~s}^{-1}\right)$. In this condition, the geometry of BP is fixed to the $\mathrm{S}_{0}$ geometry and only $\mathrm{S}_{0} @ \mathrm{~S}_{0}, \mathrm{~S}_{1} @ \mathrm{~S}_{0}$, $\mathrm{T}_{1} @ \mathrm{~S}_{0}, \mathrm{~T}_{2} @ \mathrm{~S}_{0}$, and $\mathrm{T}_{3} @ \mathrm{~S}_{0}$ are populated. Figure 3a shows the calculated populations in the time range of $0-0.5 \mathrm{~ns}$. At first, $T_{2}$ and $\mathrm{T}_{3}$ are populated via the $\mathrm{S}_{1} \rightarrow \mathrm{T}_{2}$ and $\mathrm{S}_{1} \rightarrow \mathrm{T}_{3}$ ISCs, because these ISCs are far faster than the $S_{1} \rightarrow T_{1}$ ISC and the $S_{1} \rightarrow S_{0}$ radiative and nonradiative decays (Table 1). To investigate an influence of the $S_{1} \rightarrow T_{3}$ ISC on the population dynamics, we calculated populations by setting the $\mathrm{S}_{1} \rightarrow \mathrm{T}_{3}$ ISC rate constant to be $0 \mathrm{~s}^{-1}$ (Figure $3 \mathrm{~b}$ ). We expected that $T_{3}$ would not be populated because the $S_{1} \rightarrow T_{3}$ ISC is forbidden. However, against our expectations, the $T_{3}$ population without the $S_{1} \rightarrow T_{3}$ ISC (Figure $3 b$ ) was almost as great as that obtained when fast $S_{1} \rightarrow T_{3}$ ISC was allowed (Figure $3 a$ ). This result indicates that $T_{3}$ is generated by fast $\mathrm{T}_{2} \rightarrow \mathrm{T}_{3}$ IC after $\mathrm{S}_{1} \rightarrow \mathrm{T}_{2}$ ISC. Thus, $\mathrm{T}_{3}$ is involved in the decay mechanism irrespective of the presence of the $\mathrm{S}_{1} \rightarrow \mathrm{T}_{3}$ ISC. Experimental observations of $\mathrm{T}_{3}$ would be difficult because of the similar time evolution of the populations and small $\mathrm{T}_{2}-\mathrm{T}_{3}$ energy difference of $67 \mathrm{meV}$. Improved energy resolution (for example, through the use of picosecond transient absorption spectroscopy) would enable direct detection of the simultaneous generation of $\mathrm{T}_{2}$ and $\mathrm{T}_{3}$. 
Next, $T_{1}$ is populated via the $T_{2} \rightarrow T_{1}$ IC (Figure $3 c$ ). The $T_{2}$ and $T_{3}$ populations take their maximum values of 0.43 and 0.12 , respectively, at $t \approx 0.1 \mathrm{~ns}$ and almost vanish when $t \approx 1$ $\mathrm{ns}$, whereas the $\mathrm{T}_{1}$ population takes its maximum value of 0.91 at $t \approx 0.5 \mathrm{~ns}$. After $0.2 \mathrm{~ns}$, the $\mathrm{T}_{1}$ $\rightarrow \mathrm{S}_{0}$ ISC also occurs and $\mathrm{S}_{0}$ is increased. Figure $3 \mathrm{~d}$ shows calculated populations in the time range of $0-35 \mathrm{~ns}$. After $t=1 \mathrm{~ns}$, the $\mathrm{T}_{1} \rightarrow \mathrm{S}_{0}$ ISC occurs mainly and $\mathrm{T}_{1}$ almost completely decays to $\mathrm{S}_{0}$ when $t=25 \mathrm{~ns}$ (at $t=25 \mathrm{~ns}$, the populations of $\mathrm{T}_{1}$ and $\mathrm{S}_{0}$ are 0.01 and 0.99 , respectively). Thus, without any geometry relaxation, the dominant decay paths are the indirect $S_{1} \rightarrow T_{2}(\leftrightarrow$ $\left.T_{3}\right) \rightarrow T_{1} \rightarrow S_{0}$ channels (one channel is without $T_{3}$ and the other is with $T_{3}$, both channels are shown as Type A in Figure 4). The so-called direct mechanism via the $S_{1} \rightarrow T_{1}$ ISC (rate of $3.1 \times 10^{8} \mathrm{~s}^{-1}$ ) is suppressed by the faster $\mathrm{S}_{1} \rightarrow \mathrm{T}_{2}$ ISC (rate constant is $1.5 \times 10^{10} \mathrm{~s}^{-1}$ ). 

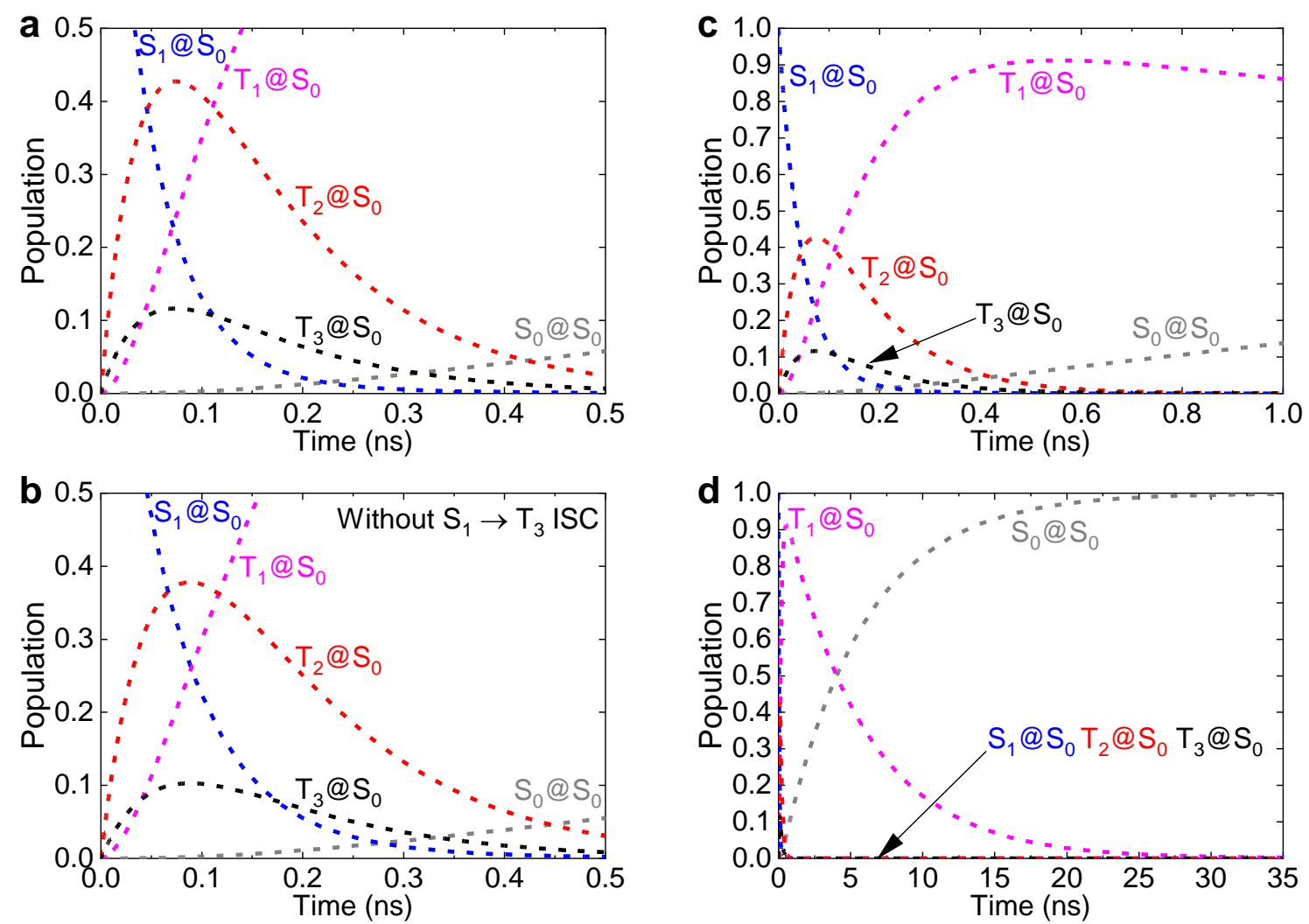

Figure 3. Calculated populations when $k_{\mathrm{GR}}=0 \mathrm{~s}^{-1}$ (without any geometry relaxation) in the time range of (a, b) 0-0.5 ns, (c) 0-1 ns, and (b) 0-35 ns. Figure 3b shows the calculated populations by setting the $S_{1} @ S_{0} \rightarrow T_{3} @ S_{0}$ rate constant to be $0 \mathrm{~s}^{-1} . \mathrm{S}_{1} @ \mathrm{~S}_{0}, \mathrm{~T}_{2} @ \mathrm{~S}_{0}$, and $\mathrm{T}_{3} @ \mathrm{~S}_{0}$ in Figure 3d almost lie on the vertical axis because they are depopulated within 1 ns (Figure 3c) and almost zero in the time range of $1-35 \mathrm{~ns}$. The decay mechanism is categorized into the Type-A mechanism in Figure 4. 
Type A

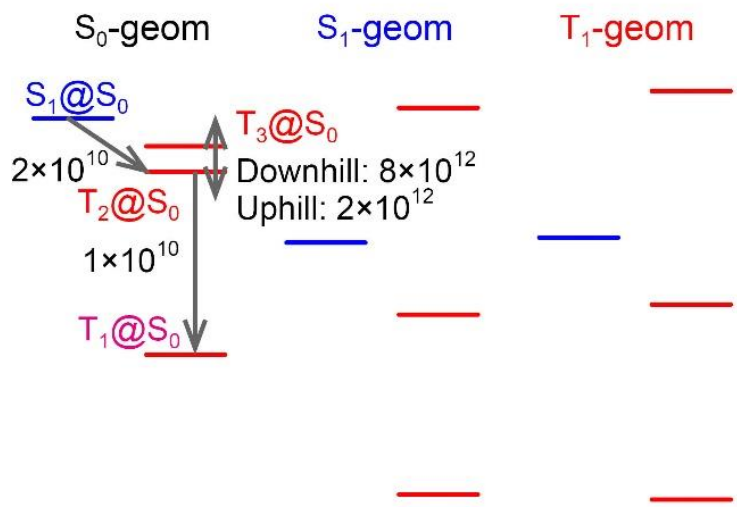

\section{Type C}

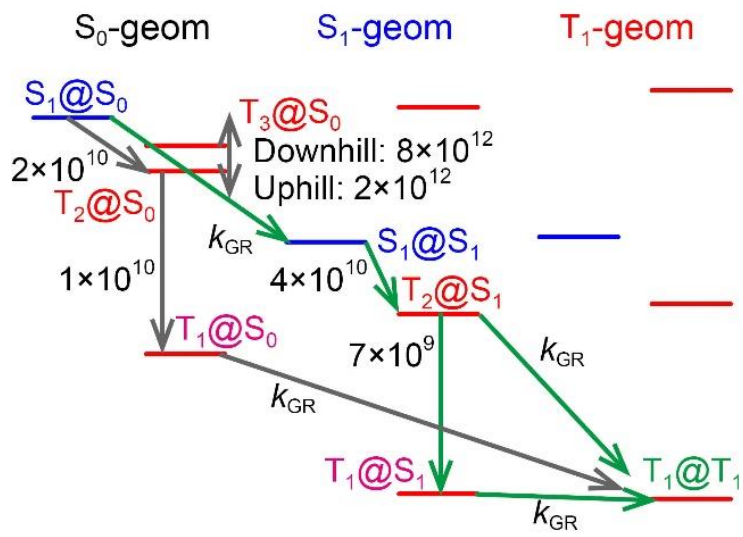

Type B

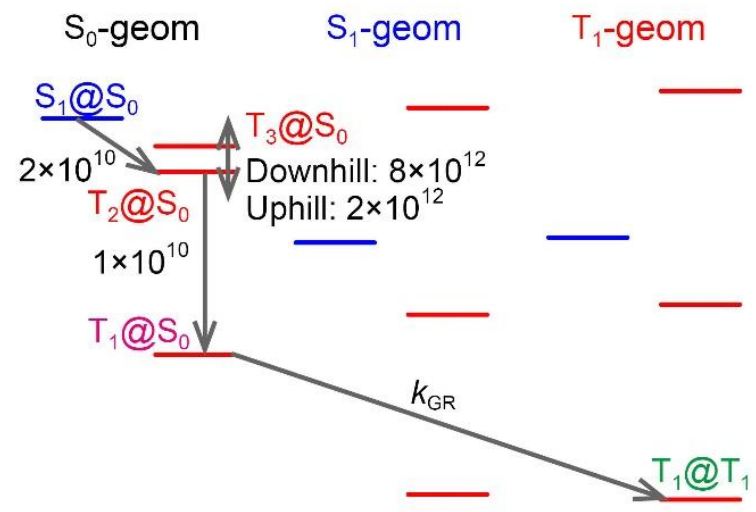

\section{Type D}

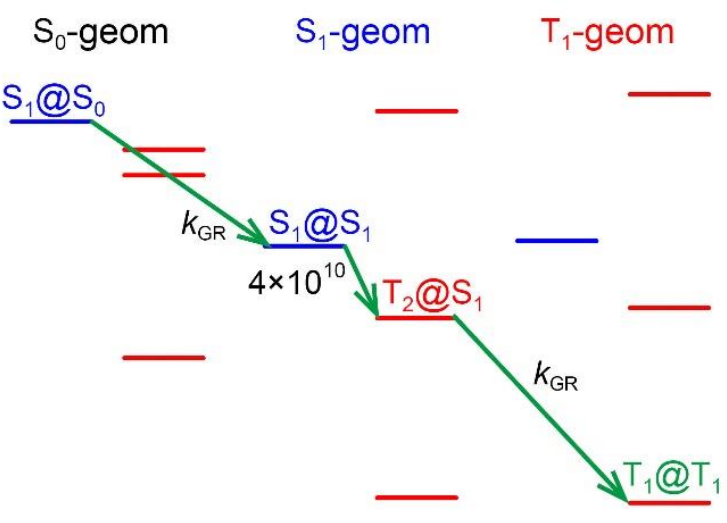

Figure 4. Dominant $T_{1}$ population mechanisms for BP: Type A, B, C, and D. Numbers are rate constants in s $\mathrm{s}^{-1} \mathrm{~T}_{2} @ \mathrm{~S}_{0}$ and $\mathrm{T}_{3} @ \mathrm{~S}_{0}$-mediated decay paths are shown by black arrows; $\mathrm{T}_{2} @ \mathrm{~S}_{1^{-}}$ mediated decay paths are shown by green arrows. 

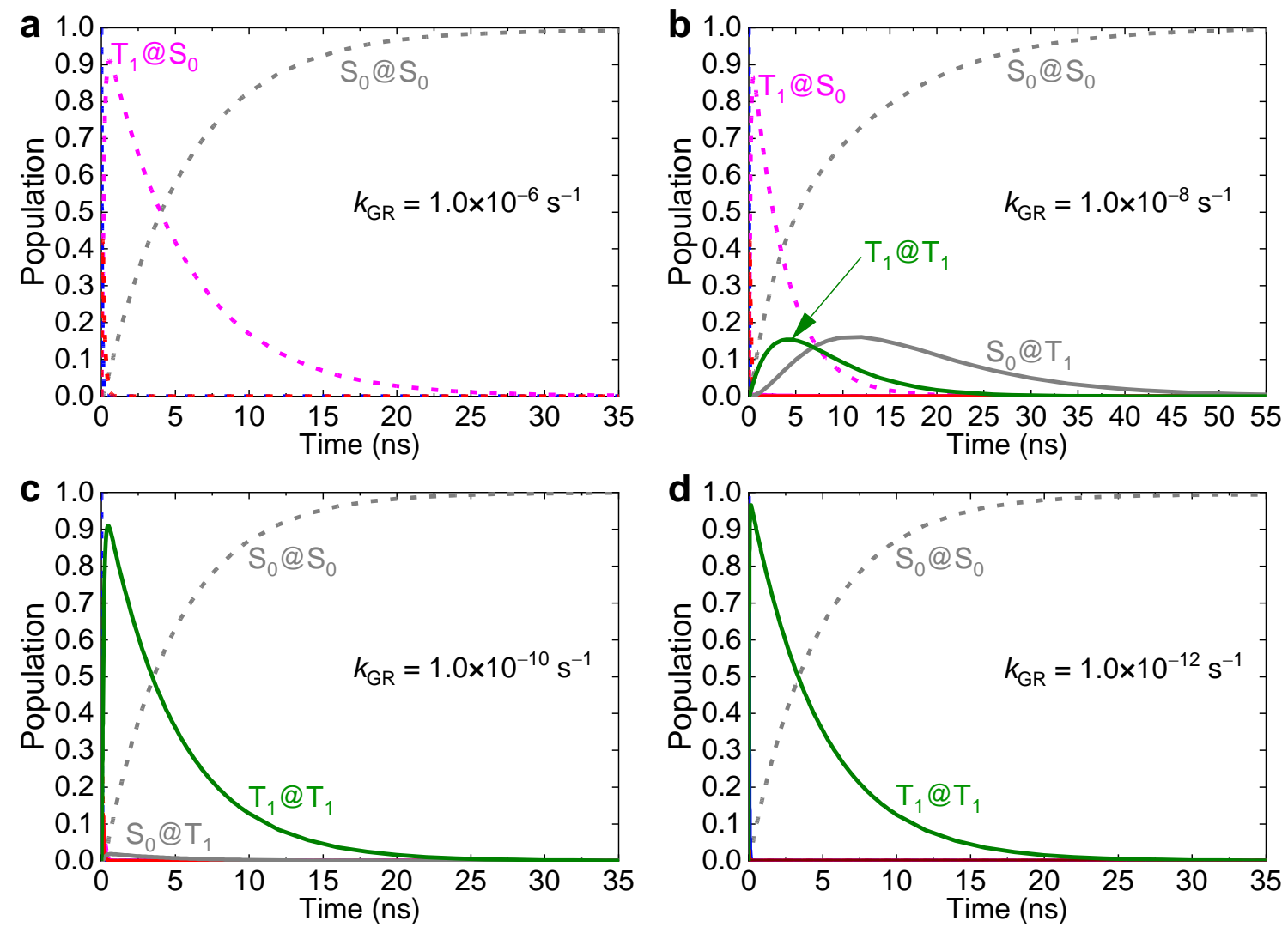

Figure 5. Calculated population dynamics when (a) $k_{\mathrm{GR}}=1.0 \times 10^{6} \mathrm{~s}^{-1}$ (Type A), (b) $k_{\mathrm{GR}}=$ $1.0 \times 10^{8} \mathrm{~s}^{-1}$ (Type B), (c) $k_{\mathrm{GR}}=1.0 \times 10^{10} \mathrm{~s}^{-1}$ (Type C), and (d) $k_{\mathrm{GR}}=1.0 \times 10^{12} \mathrm{~s}^{-1}$ (Type D). Detailed population dynamics in the time range of $0-1$ ns are reported in the Supporting Information. 
Next, we investigate decay paths considering geometry relaxation. Four cases are examined by varying the $k_{\mathrm{GR}}$ values: (1) geometry relaxations occur slower than the relevant ISCs and ICs at the $\mathrm{S}_{0}$ geometry $\left(k_{\mathrm{GR}}=1.0 \times 10^{6} \mathrm{~s}^{-1}\right)$; (2) geometry relaxations occur slower than the relevant ISCs and ICs at the $\mathrm{S}_{0}$ geometry but compete with the $\mathrm{T}_{1} \rightarrow \mathrm{S}_{0} \mathrm{ISC}\left(k_{\mathrm{GR}}=1.0 \times 10^{8}\right.$ $\left.\mathrm{s}^{-1}\right)$; (3) geometry relaxations compete with the $\mathrm{S}_{1} \rightarrow \mathrm{T}_{2}$ ISCs $\left(k_{\mathrm{GR}}=1.0 \times 10^{10} \mathrm{~s}^{-1}\right)$; (4) geometry relaxations occur faster than the relevant ISCs and ICs $\left(k_{\mathrm{GR}}=1.0 \times 10^{12} \mathrm{~s}^{-1}\right)$. From calculated population dynamics, decay pathways depend on $k_{\mathrm{GR}}$ (Figure 4).

Figure 5 shows the calculated population dynamics for the four $k_{\mathrm{GR}}$ values. The population dynamics in the short time range are reported in detail in the Supporting Information. When (1) $k_{\mathrm{GR}}=1.0 \times 10^{6} \mathrm{~s}^{-1}$, because the $\mathrm{S}_{1} @ \mathrm{~S}_{0} \rightarrow \mathrm{S}_{1} @ \mathrm{~S}_{1}$ geometry relaxation occurs much slower than the $\mathrm{S}_{1} @ \mathrm{~S}_{0} \rightarrow \mathrm{T}_{2} @ \mathrm{~S}_{0} \operatorname{ISC}\left(1.5 \times 10^{10} \mathrm{~s}^{-1}\right)$, the molecular geometry is fixed to the $\mathrm{S}_{0}$ geometry during the electronic transitions and the population dynamics (Figure 5a) are almost identical to those obtained when $k_{\mathrm{GR}}=0 \mathrm{~s}^{-1}$ (Figure 3b). Hence, the decay channel is categorized into Type A (Figure 4). When (2) $k_{\mathrm{GR}}=1.0 \times 10^{8} \mathrm{~s}^{-1}$, because the $\mathrm{T}_{1} @ \mathrm{~S}_{0} \rightarrow \mathrm{T}_{1} @ \mathrm{~T}_{1}$ geometry relaxation competes with the $\mathrm{T}_{1} @ \mathrm{~S}_{0} \rightarrow \mathrm{S}_{0} @ \mathrm{~S}_{0} \mathrm{ISC}\left(1.8 \times 10^{8} \mathrm{~s}^{-1}\right), \mathrm{S}_{0} @ \mathrm{~S}_{0}$ and $\mathrm{T}_{1} @ \mathrm{~T}_{1}$ are simultaneously populated after $\mathrm{T}_{1} @ \mathrm{~S}_{0}$ is produced (Figure $5 \mathrm{~b}$ ), suggesting that BP takes the $\mathrm{S}_{0}$ and $\mathrm{T}_{1}$ geometries and the decay channel is categorized into Type $\mathrm{B}$ (Figure 4). When (3) $k_{\mathrm{GR}}=$ $1.0 \times 10^{10} \mathrm{~s}^{-1}$, because the $\mathrm{S}_{1} @ \mathrm{~S}_{0} \rightarrow \mathrm{S}_{1} @ \mathrm{~S}_{1}$ geometry relaxation and $\mathrm{S}_{1} @ \mathrm{~S}_{0} \rightarrow \mathrm{T}_{2} @ \mathrm{~S}_{0} \mathrm{ISC}$ $\left(1.5 \times 10^{10} \mathrm{~s}^{-1}\right)$ occur in the same time scale, $\mathrm{S}_{1} @ \mathrm{~S}_{1}$ and $\mathrm{T}_{2} @ \mathrm{~S}_{0}$ first increase simultaneously. Then, the successive $\mathrm{T}_{2} @ \mathrm{~S}_{0} \rightarrow \mathrm{T}_{1} @ \mathrm{~S}_{0} \rightarrow \mathrm{T}_{1} @ \mathrm{~T}_{1}$ and $\mathrm{S}_{1} @ \mathrm{~S}_{1} \rightarrow \mathrm{T}_{2} @ \mathrm{~S}_{1} \rightarrow \mathrm{T}_{1} @ \mathrm{~S}_{1} \rightarrow \mathrm{T}_{1} @ \mathrm{~T}_{1}$ conversions predominantly occur and $\mathrm{T}_{1} @ \mathrm{~T}_{1}$ is largely populated (Figure $5 \mathrm{c}$ ). Thus, BP takes the $\mathrm{S}_{0}, \mathrm{~S}_{1}$, and $\mathrm{T}_{1}$ geometries and the decay channel is categorized into Type $\mathrm{C}$ (Figure 4). When (4) $k_{\mathrm{GR}}=1.0 \times 10^{12} \mathrm{~s}^{-1}$, because the $\mathrm{S}_{1} @ \mathrm{~S}_{0} \rightarrow \mathrm{S}_{1} @ \mathrm{~S}_{1}$ geometry relaxation occurs much faster than 
competitive ISCs at the $\mathrm{S}_{0}$ geometry $\left(3.1 \times 10^{8}-1.5 \times 10^{10} \mathrm{~s}^{-1}\right)$, the $\mathrm{S}_{1} @ \mathrm{~S}_{1}$ production is favored and $\mathrm{S}_{1} @ \mathrm{~S}_{0}$ is almost completely converted into $\mathrm{S}_{1} @ \mathrm{~S}_{1}$ within 4 ps. For the population dynamics in the time range of $0-10 \mathrm{ps}$, see Supporting Information. After $4 \mathrm{ps}$, successive $\mathrm{S}_{1} @ \mathrm{~S}_{1} \rightarrow$ $\mathrm{T}_{2} @ \mathrm{~S}_{1} \rightarrow \mathrm{T}_{1} @ \mathrm{~T}_{1}$ occurs and $\mathrm{T}_{1} @ \mathrm{~T}_{1}$ is largely populated (Figure 5d). However, unlike in case (3) $k_{\mathrm{GR}}=1.0 \times 10^{10} \mathrm{~s}^{-1}$, the $\mathrm{T}_{2} @ \mathrm{~S}_{0}$-mediated decay path is suppressed by the fast $\mathrm{S}_{1} @ \mathrm{~S}_{0} \rightarrow$ $\mathrm{S}_{1} @ \mathrm{~S}_{1}$ geometry relaxation and the decay channel is categorized into Type D (Figure 4).

The decay mechanism of photoexcited BP depends on $k_{\mathrm{GR}}$. When the GR does not occur and is slow (Type A and B), the decay path is $\mathrm{S}_{1} \rightarrow \mathrm{T}_{2} \leftrightarrow \mathrm{T}_{3} \rightarrow \mathrm{T}_{1} \rightarrow \mathrm{S}_{0}$. When the GR is intermediate (Type C), two pathways are possible, $\mathrm{S}_{1} \rightarrow \mathrm{T}_{2} \leftrightarrow \mathrm{T}_{3} \rightarrow \mathrm{T}_{1} \rightarrow \mathrm{S}_{0}$ and $\mathrm{S}_{1} \rightarrow \mathrm{T}_{2} \rightarrow \mathrm{T}_{1}$ $\rightarrow \mathrm{S}_{0}$. When the GR is fast (Type D), the path is $\mathrm{S}_{1} \rightarrow \mathrm{T}_{2} \rightarrow \mathrm{T}_{1} \rightarrow \mathrm{S}_{0}$. Therefore, we conclude that there are two kinds of decay channels for photoexcited BP; $S_{1} \rightarrow T_{2} \leftrightarrow T_{3} \rightarrow T_{1} \rightarrow S_{0}$ and/or $\mathrm{S}_{1} \rightarrow \mathrm{T}_{2} \rightarrow \mathrm{T}_{1} \rightarrow \mathrm{S}_{0}$. These are is uniformly expressed as $\mathrm{S}_{1} \rightarrow \mathrm{T}_{2}\left(\leftrightarrow \mathrm{T}_{3}\right) \rightarrow \mathrm{T}_{1} \rightarrow \mathrm{S}_{0}$. In this sense, our calculations support the experimentally proposed indirect $S_{1} \rightarrow T_{2} \rightarrow T_{1}$ decay channels ${ }^{28,30}$ among various models as described in the Introduction. This is because the distinction of $T_{2}$ and $T_{3}$ is very difficult experimentally owing to the similar time evolution of their populations (Figure 3 ) and very close $\mathrm{T}_{2}-\mathrm{T}_{3}$ energies (Types $\mathrm{A}-\mathrm{C}$ in Figure 4 ). The predicted decay mechanism is reasonably consistent with that determined by more sophisticated quantum chemical methods based on CASSCF and quantum dynamics methods in that $\mathrm{T}_{2}$ is involved as an intermediate state. ${ }^{32-33}$ In this study, we did not intended to reject the possibility of other intermediate states, such as highly excited singlet states. In our present calculations, multiphoton and multiexciton processes are not included and hence, fission of highly excited singlet states produced via biphotonic excitation ${ }^{26}$ is beyond our consideration. 


\section{SUMMARY}

We theoretically determined the excited-state decay paths of photo-excited BP and related rate constants on the basis of the cost-effective DFT and TD-DFT calculations and the Fermi golden rule. The excited-state population dynamics and resulting decay mechanism depends on the rate of geometry relaxation. When the rate of the geometry relaxation was slower than $10^{8} \mathrm{~s}^{-1}$, BP decays via the $\mathrm{T}_{2} @ \mathrm{~S}_{0}$ and $\mathrm{T}_{3} @ \mathrm{~S}_{0}$-mediated paths. In addition, when the rate of geometry relaxation is faster than $10^{10} \mathrm{~s}^{-1}$, BP takes the $\mathrm{S}_{1}$ geometry and decays via the $\mathrm{T}_{2} @ \mathrm{~S}_{1}$-mediated pathway. In any case, the dominant decay path involves the indirect $S_{1} \rightarrow T_{2} \rightarrow T_{1}$ decay channels. The predicted decay paths were consistent with the experimentally suggested one. In addition, the calculated rate constants are in excellent agreement with experimental results. Our method offers a cost-effective prediction of rate constants and excited decay pathways of molecules. These features are advantageous for theoretical design of novel optoelectronic materials. 


\section{ASSOCIATED CONTENT}

\section{Supporting Information}

Standard nuclear orientations of the optimized $\mathrm{S}_{0}, \mathrm{~S}_{1}, \mathrm{~T}_{1}$, and $\mathrm{T}_{3}$ geometries of BP. Bond lengths and the dihedral angle between the phenyl rings of the optimized $\mathrm{S}_{0}, \mathrm{~S}_{1}, \mathrm{~T}_{1}$, and $\mathrm{T}_{3}$ geometries. HOMO-4, HOMO-3, HOMO-2, HOMO-1, HOMO, LUMO, LUMO+1, LUMO+2, LUMO+3, and LUMO+4 calculated for the optimized $\mathrm{S}_{0}, \mathrm{~S}_{1}, \mathrm{~T}_{1}$, and $\mathrm{T}_{3}$ geometries. Excitation energies of $\mathrm{S}_{0}, \mathrm{~S}_{1}, \mathrm{~T}_{1}, \mathrm{~T}_{2}$, and $\mathrm{T}_{3}$ calculated at the $\mathrm{S}_{0}, \mathrm{~S}_{1}, \mathrm{~T}_{1}$, and $\mathrm{T}_{3}$ geometries. Franck-Condon energy differences between $S_{0}, S_{1}, T_{1}, T_{2}$, and $T_{3}$ calculated for the optimized $S_{0}, S_{1}, T_{1}$, and $T_{3}$ geometries. Adiabatic energy differences between $\mathrm{S}_{0}, \mathrm{~S}_{1}, \mathrm{~T}_{1}, \mathrm{~T}_{2}$, and $\mathrm{T}_{3}$. Spin-orbit couplings

$\left|\left\langle\mathrm{S}_{k}\left|\mathcal{H}_{\mathrm{SOC}}\right| \mathrm{T}_{l}^{M_{\mathrm{S}}}\right\rangle\right|$ between $\mathrm{S}_{0}, \mathrm{~S}_{1}, \mathrm{~T}_{1}, \mathrm{~T}_{2}$, and $\mathrm{T}_{3}$ calculated for the optimized $\mathrm{S}_{0}, \mathrm{~S}_{1}, \mathrm{~T}_{1}$, and $\mathrm{T}_{3}$ geometries. Population dynamics in the short time range.

\section{AUTHOR INFORMATION}

\section{Corresponding Author}

Hironori Kaji - Institute for Chemical Research, Kyoto University, Uji, Kyoto 611-0011, Japan; orcid.org/0000-0002-5111-3852; Email: kaji@scl.kyoto-u.ac.jp

\section{Authors}

Katsuyuki Shizu - Institute for Chemical Research, Kyoto University, Uji, Kyoto 611-0011, Japan; orcid.org/0000-0002-1835-0418 


\section{Author Contributions}

K.S. carried out all the theoretical calculations. H.K. planned and supervised the project. All authors contributed to the writing of this manuscript. All authors have given approval to the final version of the manuscript.

\section{Notes}

The authors declare no competing financial interest.

\section{ACKNOWLEDGMENTS}

The quantum chemical calculations using the Gaussian 16 package were performed using the SuperComputer System, Institute for Chemical Research, Kyoto University. This work was also supported by JSPS KAKENHI grant number JP20H05840 (Grant-in-Aid for Transformative Research Areas, “Dynamic Exciton”), 17H01231, 17K14529, and 19K05629. We thank Andrew Jackson, PhD, from Edanz Group (https://en-author-services.edanz.com/ac) for editing a draft of this manuscript.

\section{ABBREVIATIONS}

BP, benzophenone; fwhm, full-width at half-maximum; IC, internal conversion; ISC, intersystem crossing; RISC, reverse intersystem crossing; SOC, spin-orbit coupling. 


\section{REFERENCES}

1. Turro, N. J.; Ramamurthy, V.; Scaiano, J. C., Modern Molecular Photochemistry of Organic Molecules; University Science Books: Sausalito, 2010.

2. Parr, R. G.; Yang, W., Density Functional Theory of Atoms and Molecules; Oxford University Press: New York, 1989.

3. Runge, E.; Gross, E. K. U., Density-Functional Theory for Time-Dependent Systems. Phys. Rev. Lett. 1984, 52, 997.

4. Siegbahn, P.; Heiberg, A.; Roos, B.; Levy, B., A Comparison of the Super-Ci and the Newton-Raphson Scheme in the Complete Active Space Scf Method. Physica Scripta 1980, 21, 323-327.

5. Roos, B. O.; Taylor, P. R.; Sigbahn, P. E. M., A Complete Active Space Scf Method (Casscf) Using a Density Matrix Formulated Super-Ci Approach. Chem. Phys. 1980, 48, 157173.

6. Siegbahn, P. E. M.; Almlöf, J.; Heiberg, A.; Roos, B. O., The Complete Active Space Scf (Casscf) Method in a Newton-Raphson Formulation with Application to the Hno Molecule. $J$. Chem. Phys. 1981, 74, 2384-2396.

7. Hirao, K., Multireference Møller-Plesset Perturbation Treatment of Potential Energy Curve of N2. Int. J. Quantum Chem. 1992, 44, 517-526.

8. Hirao, K., Multireference Møller-Plesset Method. Chem. Phys. Lett. 1992, 190, 374-380.

9. Hirao, K., Multireference Møller-Plesset Perturbation Theory for High-Spin Open-Shell Systems. Chem. Phys. Lett. 1992, 196, 397-403.

10. Dirac, P. A. M., The Quantum Theory of the Emission and Absorption of Radiation.

Proceedings of the Royal Society of London. Series A 1927, 114, 243.

11. Marcus, R. A., On the Theory of Oxidation-Reduction Reactions Involving Electron Transfer. I. J. Chem. Phys. 1956, 24, 966-978.

12. Marcus, R. A., Electrostatic Free Energy and Other Properties of States Having Nonequilibrium Polarization. I. J. Chem. Phys. 1956, 24, 979-989.

13. Marcus, R. A., Electron Transfer Reactions in Chemistry. Theory and Experiment. Rev. Mod. Phys. 1993, 65, 599-610.

14. Brédas, J.-L.; Beljonne, D.; Coropceanu, V.; Cornil, J., Charge-Transfer and EnergyTransfer Processes in П-Conjugated Oligomers and Polymers: A Molecular Picture. Chem. Rev. 2004, 104, 4971-5004.

15. Samanta, P. K.; Kim, D.; Coropceanu, V.; Brédas, J.-L., Up-Conversion Intersystem Crossing Rates in Organic Emitters for Thermally Activated Delayed Fluorescence: Impact of the Nature of Singlet Vs Triplet Excited States. J. Am. Chem. Soc. 2017, 139, 4042-4051.

16. Lee, M. H.; Dunietz, B. D.; Geva, E., Calculation from First Principles of Intramolecular Golden-Rule Rate Constants for Photo-Induced Electron Transfer in Molecular Donor-Acceptor Systems. J. Phys. Chem. C 2013, 117, 23391-23401.

17. Lee, M. H.; Geva, E.; Dunietz, B. D., Calculation from First-Principles of Golden Rule Rate Constants for Photoinduced Subphthalocyanine/Fullerene Interfacial Charge Transfer and Recombination in Organic Photovoltaic Cells. J. Phys. Chem. C 2014, 118, 9780-9789.

18. Lee, M. H.; Dunietz, B. D.; Geva, E., Donor-to-Donor Vs Donor-to-Acceptor Interfacial Charge Transfer States in the Phthalocyanine-Fullerene Organic Photovoltaic System. J. Phys. Chem. Lett. 2014, 5, 3810-3816. 
19. Wilcox, D. E.; Lee, M. H.; Sykes, M. E.; Niedringhaus, A.; Geva, E.; Dunietz, B. D.; Shtein, M.; Ogilvie, J. P., Ultrafast Charge-Transfer Dynamics at the Boron Subphthalocyanine Chloride/C60 Heterojunction: Comparison between Experiment and Theory. J. Phys. Chem. Lett. 2015, 6, 569-575.

20. Gilmore, E. H.; Gibson, G. E.; McClure, D. S., Absolute Quantum Efficiencies of Luminescence of Organic Molecules in Solid Solution. J. Chem. Phys. 1952, 20, 829-836.

21. Gilmore, E. H.; Gibson, G. E.; McClure, D. S., Errata: Absolute Quantum Efficiencies of Luminescence of Organic Molecules in Solid Solution. J. Chem. Phys. 1955, 23, 399-399.

22. Batley, M.; Kearns, D. R., The Second Triplet State of Benzophenone. Chem. Phys. Lett. 1968, 2, 423-425.

23. El-Sayed, M. A.; Leyerle, R., Low Field Zeeman Effect and the Mechanism of the S1 $\rightarrow$ T1 Nonradiative Process. J. Chem. Phys. 1975, 62, 1579-1580.

24. Ohmori, N.; Suzuki, T.; Ito, M., Why Does Intersystem Crossing Occur in Isolated Molecules of Benzaldehyde, Acetophenone, and Benzophenone? J. Phys. Chem. 1988, 92, 10861093.

25. Matsushita, Y.; Kajii, Y.; Obi, K., Photochemical Reaction of Excited Benzophenone in the Gas Phase. J. Phys. Chem. 1992, 96, 4455-4458.

26. Katoh, R.; Kotani, M.; Hirata, Y.; Okada, T., Triplet Exciton Formation in a Benzophenone Single Crystal Studied by Picosecond Time-Resolved Absorption Spectroscopy. Chem. Phys. Lett. 1997, 264, 631-635.

27. Ramseier, M.; Senn, P.; Wirz, J., Photohydration of Benzophenone in Aqueous Acid. $J$. Phys. Chem. A 2003, 107, 3305-3315.

28. Yabumoto, S.; Sato, S.; Hamaguchi, H., Vibrational and Electronic Infrared Absorption Spectra of Benzophenone in the Lowest Excited Triplet State. Chem. Phys. Lett. 2005, 416, 100103.

29. Minns, R. S.; Parker, D. S. N.; Penfold, T. J.; Worth, G. A.; Fielding, H. H., Competing Ultrafast Intersystem Crossing and Internal Conversion in the "Channel 3" Region of Benzene. Phys. Chem. Chem. Phys. 2010, 12, 15607-15615.

30. Aloïse, S.; Ruckebusch, C.; Blanchet, L.; Réhault, J.; Buntinx, G.; Huvenne, J.-P., The Benzophenone S1(N, $\left.\Pi^{*}\right) \rightarrow \mathrm{T} 1\left(\mathrm{~N}, \Pi^{*}\right)$ States Intersystem Crossing Reinvestigated by Ultrafast Absorption Spectroscopy and Multivariate Curve Resolution. J. Phys. Chem. A 2008, 112, 224231.

31. Miyasaka, H.; Mataga, N., Picosecond Laser Photolysis Studies on the Photoreduction of Excited Benzophenone by Diphenylamine in Solutions. Bull. Chem. Soc. Jpn. 1990, 63, 131-137. 32. Sergentu, D.-C.; Maurice, R.; Havenith, R. W. A.; Broer, R.; Roca-Sanjuán, D., Computational Determination of the Dominant Triplet Population Mechanism in Photoexcited Benzophenone. Phys. Chem. Chem. Phys. 2014, 16, 25393-25403.

33. Marazzi, M.; Mai, S.; Roca-Sanjuán, D.; Delcey, M. G.; Lindh, R.; González, L.; Monari, A., Benzophenone Ultrafast Triplet Population: Revisiting the Kinetic Model by SurfaceHopping Dynamics. J. Phys. Chem. Lett. 2016, 7, 622-626.

34. Kubo, R., Thermal Ionization of Trapped Electrons. Phys. Rev. 1952, 86, 929.

35. Lin, S., Rate of Interconversion of Electronic and Vibrational Energy. J. Chem. Phys. 1966, $44,3759$.

36. Niu, Y.; Peng, Q.; Deng, C.; Gao, X.; Shuai, Z., Theory of Excited State Decays and Optical Spectra: Application to Polyatomic Molecules. J. Phys. Chem. A 2010, 114, 7817-7831. 
37. Shizu, K.; Adachi, C.; Kaji, H., Effect of Vibronic Coupling on Correlated Triplet Pair Formation in the Singlet Fission Process of Linked Tetracene Dimers. J. Phys. Chem. A 2020, 124, 3641-3651.

38. Shizu, K.; Adachi, C.; Kaji, H., Visual Understanding of Vibronic Coupling and Quantitative Rate Expression for Singlet Fission in Molecular Aggregates. Bull. Chem. Soc. Jpn. 2020, 93, 1305-1313.

39. Camposeo, A.; Polo, M.; Tavazzi, S.; Silvestri, L.; Spearman, P.; Cingolani, R.; Pisignano, D., Polarized Superradiance from Delocalized Exciton Transitions in Tetracene Single Crystals. Phys. Rev. B 2010, 81, 033306.

40. Kolomeisky, A. B.; Feng, X.; Krylov, A. I., A Simple Kinetic Model for Singlet Fission: A Role of Electronic and Entropic Contributions to Macroscopic Rates. J. Phys. Chem. C 2014, $118,5188-5195$.

41. Koseki, S.; Schmidt, M. W.; Gordon, M. S., MCSCF/6-31G(d,p) Calculations of OneElectron Spin-Orbit Coupling Constants in Diatomic Molecules. J. Phys. Chem. 1992, 96, 10768-10772.

42. Kim, I.; Jeon, S. O.; Jeong, D.; Choi, H.; Son, W.-J.; Kim, D.; Rhee, Y. M.; Lee, H. S., Spin-Vibronic Model for Quantitative Prediction of Reverse Intersystem Crossing Rate in Thermally Activated Delayed Fluorescence Systems. J. Chem. Theory Comput. 2020, 16, 621632.

43. Gibson, J.; Monkman, A. P.; Penfold, T. J., The Importance of Vibronic Coupling for Efficient Reverse Intersystem Crossing in Thermally Activated Delayed Fluorescence Molecules. ChemPhysChem 2016, 17, 2956-2961.

44. Minaev, B.; Minaeva, V.; Ågren, H., Theoretical Study of the Cyclometalated Iridium(Iii) Complexes Used as Chromophores for Organic Light-Emitting Diodes. J. Phys. Chem. A 2009, $113,726-735$.

45. Li, X.; Minaev, B.; Ågren, H.; Tian, H., Density Functional Theory Study of Photophysical Properties of Iridium(Iii) Complexes with Phenylisoquinoline and Phenylpyridine Ligands. $J$. Phys. Chem. C 2011, 115, 20724-20731.

46. Minaev, B.; Baryshnikov, G.; Agren, H., Principles of Phosphorescent Organic Light Emitting Devices. Phys. Chem. Chem. Phys. 2014, 16, 1719-1758.

47. Toptygin, D., Effects of the Solvent Refractive Index and Its Dispersion on the Radiative Decay Rate and Extinction Coefficient of a Fluorescent Solute. J. Fluoresc. 2003, 13, 201-219. 48. El-Sayed, M. A., Triplet State. Its Radiative and Nonradiative Properties. Acc. Chem. Res. 1968, $1,8-16$. 
TOC graphic

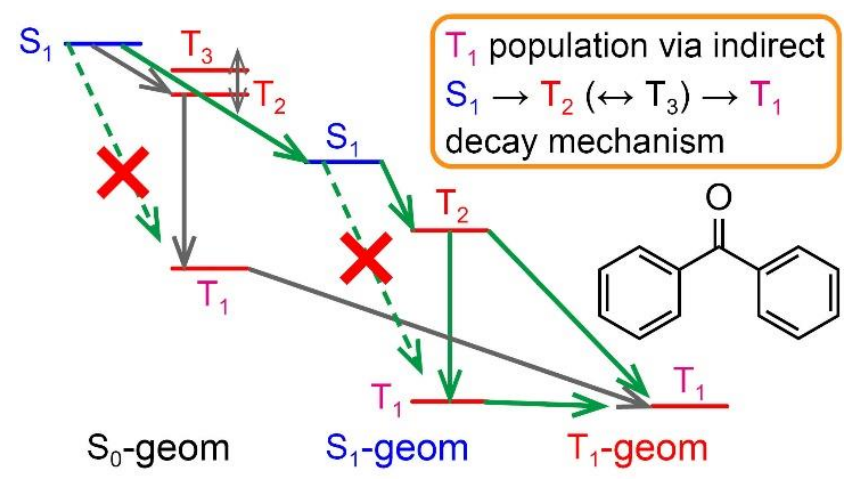

Brief description for TOC graphic

$\mathrm{S}_{1} \rightarrow \mathrm{T}_{2}\left(\leftrightarrow \mathrm{T}_{3}\right) \rightarrow \mathrm{T}_{1} \rightarrow \mathrm{S}_{0}$ is the predominant decay pathway of benzophenone. 